Les débuts de la ligne ferroviaire de Saint-Étienne à Lyon et les événements de 1830

\title{
Michel Cotte
}

\section{(2) OpenEdition}

\section{Journals}

Édition électronique

URL : https://journals.openedition.org/rhcf/1998

DOI : 10.4000/rhcf.1998

\section{Éditeur}

Rails \& histoire

\section{Édition imprimée}

Date de publication : 1 février 2003

Pagination : 218-228

ISBN : 0996-9403

ISSN : 0996-9403

\section{Référence électronique}

Michel Cotte, « Les débuts de la ligne ferroviaire de Saint-Étienne à Lyon et les événements de 1830 », Revue d'histoire des chemins de fer [En ligne], 26 | 2003, mis en ligne le 22 janvier 2015, consulté le 22 avril 2022. URL : http://journals.openedition.org/rhcf/1998 ; DOI : https://doi.org/10.4000/rhcf.1998 


\section{Les débuts de la ligne ferroviaire de Saint- Étienne à Lyon et les événements de 1830}

\section{Un beau projet non dénué de difficultés}

En mars 1826, les frères Seguin d'Annonay ${ }^{1}$ obtiennent l'adjudication de la ligne de chemin de fer devant relier le bassin minier de Saint-Étienne à la vallée du Rhône. Dans cette affaire de construction ferroviaire, très nouvelle en France ${ }^{2}$, ils sont associés à Édouard Biot, le fils du physicien et neveu de Barnabé Brisson, le principal collaborateur technique du directeur général des Ponts et Chaussées ${ }^{3}$. Le tracé est profondément novateur, il doit en particulier permettre l'usage des machines locomotives sur toute l'étendue de la ligne, soit une soixantaine de kilomètres ${ }^{4}$. D'importants travaux de génie civil sont rendus nécessaires par l'application des principes ferroviaires retenus : de longues pentes régulières et de grands rayons de courbure. De nombreux tunnels, d'importants remblais et déblais, des ouvrages d'art et de soutènement de la ligne sont nécessaires. Le projet apparaît comme ambitieux ; il doit franchir un important dénivelé, impossible à vaincre par un canal, pour désenclaver le bassin minier de Saint-Étienne ${ }^{5}$.

Le projet économique et industriel repose sur l'espoir d'un transport massif et peu cher de la houille, qui doit permettre un décollage industriel régional, à l'anglaise. Le charbon de terre était déjà familier dans la région, provenant des mines de Rive-de-Gier déjà reliées au Rhône par le très rentable canal de Givors, ouvert depuis 1780.

Abréviations : A. d. A. : Archives départementales de l'Ardèche ; C. Int. : Correspondance interne des entreprises Seguin ; S. A. : Seguin Aîné (Marc), encore dit Seguin ou l'Aîné.

1- Il s'agit de Marc, Camille, Paul et Charles ; Jules a été écarté du chemin de fer.

2- Cette ligne est la seconde construite en France, après celle de Beaunier reliant Saint-Étienne à Andrézieux sur la Loire $(18 \mathrm{~km})$. Les deux projets sont complémentaires, mais de nature et de difficulté bien différentes.

3- Becquey durant la Restauration.

4- Elle est comparable à la célèbre ligne de Liverpool à Manchester qui se construit au même moment.

5- Le bassin houiller de Saint-Étienne apparait alors comme le plus important en France. 
Parallèlement, l'effort d'adaptation des techniques anglaises et l'innovation sont poussés à un degré maximum dans ce projet, en particulier par l'ouverture d'un atelier de construction de machines locomotives dans la presqu'île de Perrache, dès 1828-1829 . Les frères Seguin, tout particulièrement leur ainé Marc, apparaissent alors au sommet de leurs talents d'entrepreneurs. Ils ont déjà à leur actif la réalisation des ponts suspendus par câbles de fil de fer (1821-1825) ; l'utilisation des machines à vapeur haute pression pour la navigation (1825-1828) ; la mise au point de la chaudière tubulaire (1827-1829).

Ces auspices heureux ne doivent cependant pas masquer les nombreuses difficultés techniques et financières rencontrées par la société anonyme créée à la suite de l'adjudication. Malgré un capital social de dix millions de francs, qui frappe les imaginations, l'importance du génie civil et la spéculation foncière grèvent les comptes de la société anonyme à partir de 1829. Un choix d'achèvement partiel de la ligne est alors décidé, visant à concurrencer directement le canal de Givors. L'avancement des travaux permet l'ouverture d'une section d'un peu plus de 20 kilomètres dès l'été 1830, allant de la mine de la GrandCroix à Givors. Dans la cité rhodanienne, la ligne se poursuit jusqu'au fleuve où une installation provisoire de la « gare d'eau » permet d'accueillir une douzaine de bateaux.

Aux difficultés techniques et financières viennent s'ajouter une agitation sociale qui débute dès la mise en service, début juillet 1830 , formant un mouvement annonciateur des journées révolutionnaires. À cette occasion, la ligne de chemin de fer devient le théâtre d'événements sociaux importants, qui anticipent et dépassent les seuls aspects politiques du changement de régime. Une sensibilité ouvrière se dégage, marquée de corporatisme, d'hostilité à la mécanisation, mais aussi d'un sens aigu de la négociation et de l'adaptation professionnelle. En face, les entrepreneurs Seguin font montre d'une perception poussée des situations sociales, tout en prenant de nombreuses initiatives patronales aux facettes les plus variées...

\section{La mise en service de la partie achevée (juin-juillet} 1830)

La mise en exploitation de la portion achevée est précédée de tests sur les deux machines locomotives sorties des ateliers. Ces expériences se déroulent d'abord sur un embranchement d'essai à

6- Il s'agit de produire une machine originale spécialisée dans le transport lourd sur des pentes régulières et importantes,(6 pour 1000 de Givors à Rive-de-Gier et 13,6 pour 1000 de cette ville à Saint-Etienne). 
Perrache, comportant une courte réplique des pentes. Après le transport d'une première machine à Givors ${ }^{7}$, Marc et Paul poursuivent les expériences sur la ligne elle-même, non sans connaitre un premier accident ferroviaire!

« Nous essayâmes hier la machine locomotive qui fonctionna très bien jusqu'à Saint-Lazare. Paul éprouva à sa jambe gauche un petit accident occasionné par cette machine. Le chariot de l'eau et du charbon sur lequel nous étions [...] à la descente, fut sorti avec violence de dessus les rails et jeté de côté. En sautant à terre, Paul eut la jambe froissée 8 .»

le $1^{\text {er }}$ juillet :

La mise en exploitation commerciale est finalement prévue pour

" [Marc et Camille] ont fait descendre deux convois chargés qui ont bien fait leur route. Il y a cependant quelques rails qui ont besoin d'être replacés?.»

Techniquement les choses s'améliorent :

«Tous les jours nos transports augmentent sur le chemin de fer. Les chariots ne sortent plus de la voie. Cependant nous n'avons pas encore mis en mouvement la machine ${ }^{10}$. »

Les premières modalités de l'exploitation se précisent entre les frères Seguin : Paul s'occupe du matériel roulant et du mouvement; Marc à Perrache monte les wagons et poursuit la construction des machines locomotives ; Camille s'installe à Rive-de-Gier pour l'organisation du trafic et les relations commerciales; Charles dirige le bureau parisien des Seguin. Un projet concernant les personnels de l'exploitation est présenté au conseil d'administration de la compagnie au moment de l'ouverture ${ }^{11}$.

Malgré une apparente satisfaction des frères durant les premiers essais d'exploitation, le passage à la traction locomotive s'avère relativement problématique et l'on utilise provisoirement les chevaux pour la remonte des wagons vides. Comme prévu, la gravité parait suffisante pour la descente des wagons de charbon, par l'utilisation d'une pente régulière sur l'ensemble de la partie livrée.

7- A. d. A. 41J/194, C. Int. de S. A. du 16 juin 1830.

8- A. d. A. 41J/194, C. Int. de S. A. du 20 juin 1830.

9- A. d. A. 41J/194, C. Int. de Paul à Lyon du $1^{\text {er }}$ juillet 1830.

10- A. d. A. 41J/194, C. Int. de Paul.

11- A. d. A. 41J/200, S. A. «Projet d'organisation par MM. les gérants », 29 juin 1830. 
Le trafic commence, nous dit Camille, dans le désordre de la mise en mouvement d'une grande affaire ${ }^{12}$. Les tarifs d'usage sont fixés à l'hectolitre (une benne), la mesure traditionnelle de capacité des exploitants houillers. Ils sont fixés à 14 centimes au départ de Rive-de-Gier et à 21,25 centimes à celui de la Grand-Croix ${ }^{13}$. Ce qui revient à une petite majoration de distance, surtout dans le second cas, par rapport à un tarif d'adjudication de 9,8 centimes la tonne/kilomètre.

Du point de vue de la compagnie, qui pourrait exiger tout de suite un droit de regard sur l'exploitation des frères, les choses se passent assez amicalement grâce à la compréhension de Humblot-Conté. Chargé par le conseil d'administration d'examiner cette première mise en fonctionnement, il accepte un report de l'examen des comptes jusqu'à la livraison définitive, ce qui en pratique laisse les Seguin maîtres d'organiser l'exploitation à leur guise ${ }^{14}$.

Dès la nouvelle connue de l'ouverture de la ligne, le préfet demande à la visiter, ce qui se transforme en une inauguration officieuse. Le grand tunnel de Couzon est en particulier illuminé à cette occasion et un trafic exceptionnel de 6 ou 8 convois précède le cortège des notables, à la descente sur Givors ${ }^{15}$.

\section{Le mouvement des crocheteurs, les événements de Juillet 1830}

Camille constate rapidement des progrès dans l'organisation des convois et la régularité du mouvement, mais aussi l'apparition d'une réaction sociale à la mise en exploitation du chemin de fer. La première et la plus durable vient des crocheteurs, à propos du débarquement automatisé du charbon prévu à la gare d'eau de Givors. L'ouverture d'une trappe installée dans le fond du wagon le vide dans le bateau, sans déchargement manuel des crocheteurs.

«Notre service prend tous les jours plus de consistance, aujourd'hui nous avons fait partir 80 wagons. Mais nous marchons toujours au milieu des obstacles et des oppositions de toutes espèces. Hier ça a été une révolte des crocheteurs de Givors qui se sont opposés au débarquement direct des wagons dans le Rhône par les estacades ${ }^{16}$.»

12- A. d. A. 41J/194, C. Int. de Camille du $1^{\text {er }}$ juillet 1830.

13- A. d. A. 41J/194, C. Int. de Paul du 14 juillet 1830 ; la benne contient ici 80$85 \mathrm{~kg}$.

14- A. d. A. 41J/194, C. Int. du 22 juillet 1830.

15- A. d. A. 41J/194, C. Int. des 12, 14 et 15 juillet 1830, le voyage du préfet s'est déroulé le 15 juillet.

16- A. d. A. 41J/194, C. Int. de Camille à Rive-de-Gier du 8 juillet 1830. 
Quelques jours après, les frères apprennent directement par le préfet une menace de malveillance :

«M. Desbrosses nous écrit qu'il a été informé qu'il se formait une coalition de voituriers ayant pour but de bouleverser nos rails et interrompre le service du chemin de fer $^{17}$.»

Marc est aussi victime d'intimidations sur sa personne, mais il en minimise les conséquences dans ses souvenirs :

"Quelques voituriers par terre ont quelquefois menacé de me tuer, mais jamais il n'y eut aucun commencement de tentative pour mettre ces menaces en exécution; tout s'est borné dans le début à embarrasser les rails avec des pierres, des traverses, etc., ce qui occasionna quelques accidents au matériel, mais jamais aux voyageurs $^{18}$.»

L'exploitation du chemin de fer commence plutôt laborieusement, dans le cadre d'une période sociale agitée qui débouche sur les journées révolutionnaires de Juillet 1830. Les frères nous donnent quelques indications sur les événements en région lyonnaise ; événements qui les inquiètent car ils doivent eux-mêmes faire face à une opposition corporative au chemin de fer depuis plusieurs jours.

Dans Lyon, le premier mouvement populaire semble commencer le 30 juillet au soir ${ }^{19}$; il se poursuit le lendemain matin, sans violence. L'organisation de la Garde nationale rassure les entrepreneurs ${ }^{20}$, d'autant que, le $1^{\text {er }}$ août, les prisonniers de Saint-Joseph au début de la presqu'île de Perrache tentent de s'échapper en attaquant les soldats qui les gardent. Ceux-ci réagissent violemment :

« [lls ont alors ouvert] un feu vif et soutenu qui en a tué 5 ou 6 et blessé un plus grand nombre ${ }^{21}$.»

L'effervescence gagne les ouvriers lyonnais, ceux des chantiers de Perrache en particulier.

« [...] ici toutes les têtes sont en fermentation. De toute part les ouvriers désertent les ateliers. Nos manœuvres du pont de la Mulatière ne veulent ni travailler ni sortir des chantiers ${ }^{22}$.»

17- A. d. A. 41J/194, C. Int. de Paul du 23 juillet 1830.

18- Fonds privé [Marc Seguin], «Historique du chemin de fer de St-Étienne à Lyon », ms., 12 mai 1836, fo 48.

19- Rappelons que les «Trois Glorieuses » se déroulent les 27, 28 et 29 juillet 1830 à Paris.

20- A. d. A. 41J/194, C. Int. de S. A. du 31 juillet 1830.

21- A. d. A. 41J/194, C. Int. de S. A. à Lyon du $1^{\text {er }}$ août 1830.

22- A. d. A. 41J/194, C. Int. de S. A. du 2 août 1830. 
À Givors, à Rive-de-Gier, le trafic se désorganise. Les Seguin ne veulent prendre aucun risque, craignant la virulence des crocheteurs et des voituriers. Ils songent en premier lieu à protéger le matériel roulant.

«Suivant les circonstances, vous pourrez pendant la nuit faire filer tous les wagons dans le grand percement [tunnel de Couzon] et en fermer les entrées par de bonnes barricades ${ }^{23}$.»

La rumeur circule à Lyon d'un mouvement Ultra royaliste dans le Midi, ainsi que celle de la radicalisation des événements parisiens...

Le mouvement d'opposition sociale au chemin de fer s'étend le long de la ligne ; il touche l'ensemble des crocheteurs, puis les voituriers de Givors refusent de tracter les wagons au moyen de leurs chevaux ${ }^{24}$.

« Notre mouvement insurrectionnel de la Grand-Croix n'a pas eu de suite, et les crocheteurs sont rentrés sans avoir exécuté leur projet de démolition. Mais, à Givors, nous sommes toujours sous l'empire de cette canaille ${ }^{25}$.»

Marc ordonne l'arrêt du trafic jusqu'à nouvel ordre ; il pense qu'il vaut mieux accréditer l'idée que leur affaire ne marche pas plutôt que d'en faire un pôle de violence révolutionnaire.

\section{Une situation tendue s'installe}

Toutefois, par le biais de Paul, les Seguin entrent rapidement en négociation à Givors, le lieu névralgique de la contestation. Les deux points à l'ordre du jour sont l'abandon des estacades de déchargement automatique et les salaires.

«Les crocheteurs ont profité des troubles politiques pour se faire augmenter. Nous nous en débarrasserons aussitôt que l'ordre sera rétabli ${ }^{26} . »$

En fin de semaine, le calme semble revenir dans les rangs des crocheteurs. Les frères espèrent de leur côté dans l'instauration de la garde nationale, en particulier à Givors, pour ramener le calme dans les esprits. C'est à ce moment-là que Camille songe à mettre en pratique une idée aussi simple que géniale afin de rendre populaire le chemin de fer:

\section{3- Ibid.}

24- A. d. A. 41J/194, C. Int. de Paul à Givors du 5 août 1830.

25- A. d. A. 41J/194, C. Int. de Camille à Rive-de-Gier du 5 août 1830.

26- A. d. A. 41J/194, C. Int. de Paul à Givors du 8 août 1830. 
«Rien de nouveau ici, nous continuons à travailler peu, ce qui nous a décidé à faire afficher que nous conduirions les amateurs à la vogue de Givors aujourd'hui dimanche ${ }^{27}$. »

Un trafic inopiné des voyageurs commence là, apparemment gratuit, à propos d'une fête populaire et favorisé par les troubles de 1830 ! Il semble connaitre un succès notable, contribuant à un début d'isolement social du mouvement des crocheteurs et des voituriers. Ce trafic des personnes parait se prolonger par un usage plus ou moins toléré, volontiers ludique : les jeunes gens s'accrochent aux wagons de charbon pour descendre à Givors! On peut dire que cette situation particulière a entraîné une première demande de transport des personnes et qu'elle s'est ensuite organisée et codifiée, tout en devenant payante, alors qu'elle ne faisait pas du tout partie des objectifs initiaux de la compagnie.

Les difficultés sociales n'en demeurent pas moins, pendant pratiquement tout l'été et une partie de l'automne. Complètement impliqués dans le mouvement de mécanisation des productions depuis de nombreuses années, les Seguin soulignent l'hostilité populaire à son égard :

«Les journaux d'aujourd'hui annoncent que le peuple de Paris s'est encore ameuté en demandant l'abolition des mécaniques et la suppression d'une partie des impôts ${ }^{28}$.»

« Nos ouvriers papetiers [d'Annonay] ont voulu faire hier un petit mouvement contre les machines semblable à celui des crocheteurs contre les estacades, mais notre garde nationale qui ne pense pas comme celle de Givors a pris les armes et tout est rentré dans le devoir ${ }^{29}$.»

Le ton monte, et un cycle d'intimidations réciproques s'installe à la fin du mois d'août. Les Seguin tentent cependant de reprendre les transports et leurs essais de locomotive. Le trafic semble perturbé assez gravement à plusieurs reprises.

L'appel aux nouvelles autorités de Lyon montre l'ambiguiité de la situation. Les frères sont assez mal reçus, l'Aîné des Seguin étant considéré comme un partisan du régime défunt ; toutefois ils obtiennent l'aide de la force publique.

27- A. d. A. 41J/194, C. Int. de Camille à Rive-de-Gier du 8 août 1830 ; la vogue est une grande fête populaire à date fixe.

28- A. d. A. 41J/194, C. Int. de Paul à Givors du 18 août 1830.

29- A. d. A. 41J/194, C. Int. de Camille à Annonay du 24 août 1830. 
« [lls] nous conseillent tous de filer doux, cependant le préfet a promis de doubler les postes de gendarmerie ${ }^{30}$. »

Si Marc peut être considéré comme un proche de la Restauration et un catholique convaincu, la situation d'ensemble des Seguin est beaucoup plus nuancée. Le père et les frères ont longtemps fréquenté les cercles libéraux d'Annonay. La famille lisait la presse d'opposition, affichant de surcroît une certaine impertinence à l'égard du clergé. De son côté, Charles apparait comme un proche du duc d'Orléans et un ami de son aide de camp, Rumigny, un associé des Seguin dans l'affaire de la gare d'eau de Perrache... Au moment des événements de Juillet, Charles dirige même la construction d'une passerelle suspendue dans la propriété du nouveau souverain, à Neuilly, et le rencontre presque quotidiennement. Marc évoque la complexité du retournement de situation, non sans quelque humour :

« Je ne sais pas si notre réputation plus que douteuse de francs libéraux m’avait précédé [...]. Je ne suis plus habitué [aux manières populaires] ayant beaucoup plus fait usage des formes des gens de cour qui me plaisent davantage ${ }^{31}$. »

Évidement, les Seguin ne sont pas hommes à baisser les bras devant une situation sociale difficile. Paul essaye par exemple de monter une équipe de crocheteurs dévoués au chemin de fer pour contrer ceux du canal de Givors, à la base des mouvements contre la compagnie. Si d'un côté les frères savent tout de suite négocier, ils n'hésitent pas à recourir parallèlement à la force publique et à la justice pour des actions violentes à l'encontre du chemin de fer et de ses employés. Ils s'estiment insuffisamment soutenus par les autorités nouvelles pendant un long moment; à la fin du mois d'août, ils obtiennent cependant l'aide d'une quinzaine de gendarmes ${ }^{32}$.

«Le préfet [...] s'étant décidé à nous donner main forte, on fit saisir six [crocheteurs] par la gendarmerie, qui furent promptement assouplis par le régime de la prison ${ }^{33}$. »

De son côté, Paul prévoit une surveillance dissuasive de la ligne :

"Je suis d'avis de ne pas perdre de temps pour organiser et armer nos gardes. Je vais à Lyon, j'apporterai des sabres et la commission [autorisation] ${ }^{34}$.»

30- A. d. A. 41J/194, C. Int. de Paul du 20 août 1830.

31- A. d. A. 41J/194, C. Int. de S. A. du 9 août 1830.

32- A. d. A. 41J/194, C. Int. des 26 et 27 août 1827.

33- S. A., Historique... cité, fo 49.

34- A. d. A. 41J/194, C. Int. de Paul du 18 août 1830. 
Il entend obtenir les mêmes avantages du département de la Loire et il passe commande d'une vingtaine de fusils à Saint-Étienne ${ }^{35}$. Les esprits sont réellement très échauffés à la mi-août :

«Je vais faire en sorte de vous obtenir les deux pièces de canon que vous désirez, Rumigny est bien en position de nous les faire obtenir par le général Gérard ministre de la Guerre ${ }^{36} . »$

Cette extrémité, envisagée dans les moments les plus vifs des événements, n'aboutira fort heureusement pas, mais la compagnie obtient d'avoir en permanence des gardes armés le long de la ligne. Une fois le calme social revenu, elle défendra de manière active ses intérêts matériels, basculant à l'occasion dans le drame d'un fait divers :

" [Nous avons reçu une lettre des Seguin] relative au garde Noël, condamné à une année de détention par suite d'un meurtre commis involontairement sur un individu qu'il avait surpris volant des cuivres enlevés à une machine de la Compagnie à Givors ${ }^{37}$. »

On peut penser que l'amertume de l'échec d'une partie des crocheteurs favorisa un glissement vers des gestes individuels de vandalisme ou de chapardage.

\section{Règlement du conflit des crocheteurs et mutations professionnelles}

Les événements de l'été 1830 marquent fortement l'histoire des débuts de l'exploitation de la ligne de Saint-Étienne à Lyon. En ce qui concerne le mouvement des crocheteurs, Paul poursuit ses efforts pour créer une compagnie de manutentionnaires à la seule dévotion du chemin de fer : à terme une soixantaine d'hommes assez bien payés sur la base d'un tarif à la tâche garanti par une charte. Dans le cours de l'automne, il parvient à mettre sur pied un premier groupe, à Givors, le lieu par excellence de la contestation sociale du chemin de $\mathrm{fer}^{38}$. Il s'agit de le faire reconnaitre officiellement, face à celui existant pour le canal et prétendant au monopole ${ }^{39}$. La mise sur pied d'une telle organisation n'est pas des plus tranquilles : la négociation sur les tarifs est particulièrement âpre. L'un des enjeux de la confrontation est de pouvoir contrôler

35- Ibid. et 29 août 1830 ; demande d'autorisation.

36- A. d. A. 41J/195, C. Int. de Charles à Paris du 15 août 1830.

37- A. d. A. 41J/147, séance du conseil d'administration du 7 février 1832.

38- A. d. A. 41J/194, C. Int. de Paul du 29 novembre 1830.

39- A. d. A. 41J/194, C. Int. de la seconde quinzaine d'août 1830 ; A.N. $F^{14} 9031$, pétition des crocheteurs du chemin de fer contre ceux du canal qui prétendent à l'exclusivité à Rive-de-Gier et Givors, 9 décembre 1831. 
les hommes qui vont venir travailler chez eux ; ensuite, elle transforme une contestation frontale contre le chemin de fer en une discussion interne à la profession, aboutissant à d'inévitables fractures entre ceux liés au canal et au fleuve et ceux attachés maintenant aux Seguin...

Mais le problème n'a-t-il pas simplement été repoussé dans le temps par l'urgence de la situation et la force du mouvement corporatiste ? L'emprise des crocheteurs sur la ligne de chemin de fer est momentanément reconnue, bien réelle, créant des droits.

« Les crocheteurs, à la suite des troubles de Juillet [...] s'emparèrent du chemin de fer pendant plus de trois mois; ils déchargeaient à bras d'homme les charbons des wagons dans les bateaux $[\ldots]$; cette manœuvre était très longue ${ }^{40}$. »

Dans un premier temps, cela empêche un véritable départ du transport des houilles. Quand, fin septembre, les frères tentent de reprendre l'usage des couloirs de déchargement automatique, à Givors, la réaction populaire est immédiate :

«Lorsqu'on a fait la démonstration d'avancer un wagon, les femmes sont arrivées toutes ivres [de rage] avec des pierres dans leurs tabliers et suivies des hommes. On a dit beaucoup d'injures, etc., mais point de coups ${ }^{41}$. »

Finalement les accords entre les Seguin et les crocheteurs s'élargissent, aboutissant à la prise en compte des nouveaux procédés mécaniques de déchargement par les ouvriers, contre des avantages financiers :

« Il leur est payé actuellement [35] centimes pour chaque wagon déchargé au couloir, [50] centimes à la bascule et 1 franc à bras d'homme $^{42}$.»

Grâce à leur lutte sociale et à leur esprit de revendication, les crocheteurs gagnent par ailleurs un droit prioritaire à l'embauche pour toutes les opérations de manutention le long de la ligne. Ces dispositions tendent à les transformer en des employés de la compagnie aux points de chargement, en leur octroyant une bonne prime sur l'usage des nouveaux moyens mécanisés, maintenant confiés à leur surveillance. La mutation professionnelle paraît là assez brusque, changeant en particulier la nature du travail et transformant une corporation de type ancien en un groupe

40- S. A., Historique... cité, fo 48.

41- A. d. A. 41J/194, C. Int. de Paul, [25 - 28 septembre 1830].

42- S. A., Historique... cité, fo 49 ; la bascule est un autre dispositif automatisé. 
privilégié d'ouvriers de la compagnie ferroviaire. Une hiérarchie nouvelle se crée, entre ceux qui travaillent aux dispositifs mécanisés, bien payés, et ceux qui continuent à décharger ou charger manuellement.

Sans doute onéreuses au début, ces dispositions longuement négociées ${ }^{43}$ ne sont pas qu'une contrainte pour la société du chemin de fer. Elles parviennent d'abord à régler un conflit social grave et à éloigner la menace de représailles sur les installations de la compagnie. L'augmentation du trafic permet ensuite de résorber assez vite le nombre de crocheteurs menacés par le chômage. Le cadre général de la négociation permet aux Seguin d'inclure une contrainte de travail, à leurs yeux essentielle pour l'exploitation intensive à venir :

"Il faut obtenir que le trafic se fasse de nuit et dimanche et fêtes ${ }^{44}$.»

Enfin, l'apparition, sous la contrainte, d'un droit de manutention supplémentaire au strict tarif de l'adjudication constitue un précédent intéressant pour la compagnie. Cela lui permet d'introduire des frais additionnels au transport. Elle ne se privera pas d'en ajouter plusieurs autres, jusqu'à soulever une nouvelle contestation sociale à la suite de l'ouverture générale de la ligne en 1833, celle des exploitants houillers de Saint-Étienne!

43- A. d. A. 41J/194, C. Int. jusqu'en octobre 1830.

44- A. d. A. 41J/194, C. Int. de Paul du 3 octobre 1830. 